\title{
Electron Microscopy Study of Exotic Nanostructures of Cadmium Sulfide Prepared by Catalytic Thermal Evaporation
}

\author{
Lifeng Dong and Jun Jiao \\ Department of Physics, Portland State University, Portland, OR 97201, USA
}

$\mathrm{CdS}$, one of the most important II-VI group semiconductors, is a vital optoelectronic material. Nanoscale CdS structures such as nanowires have shown potential as building blocks for optoelectronic nanodevices. For instance, single CdS nanowire electrically driven lasers have been demonstrated [1]. Recently, we have synthesized, for the first time, CdS nanobelts [2] and other types of exotic nanostructures using a thermal evaporation method. In this report, we present the results of the electron microscopy characterization of these nanomaterials. It is expected that a systematic investigation of correlations between the preparation conditions and the formation of different nanostructures will lead us to understand growth mechanisms. Eventually, this will allow growing nanostructures with controlled properties.

Our method for synthesizing CdS nanowires and nanobelts is based on the physical evaporation of CdS powder with the presence of a Au catalyst. In a quartz tube furnace in which the chamber was pumped to a vacuum of $10^{-3}$ Torr, $\mathrm{CdS}$ nanostructures were formed on the surface of Au-coated $\mathrm{W}$ substrates by heating (up to $800{ }^{\circ} \mathrm{C}$ ) $\mathrm{CdS}$ powder at 150 Torr with a constant flow of argon for 60 minutes. TEM specimens were made by carefully scratching off the formations from the $\mathrm{W}$ substrates and ultrasonicating them in ethanol. Several drops of suspension were then transferred onto the TEM grids. Electron microscopy characterizations were performed on an FEI 611 focus ion beam (FIB) microscope operated at the secondary electron mode, and a Tecnai F-20 field emission high resolution TEM/STEM/EDX at $200 \mathrm{kV}$.

Fig. 1a demonstrates that $\mathrm{CdS}$ nanowires originated only from the areas coated with Au catalyst with a required growth temperature of $500^{\circ} \mathrm{C} \sim 600{ }^{\circ} \mathrm{C}$. Fig. $1 \mathrm{~b}$ reveals a $\mathrm{Au}$ particle located at the tip of a $\mathrm{CdS}$ nanowire, which suggests a Vapor-Liquid-Solid (VLS) growth mechanism [3,4]. HRTEM characterization indicates that both the $\mathrm{CdS}$ nanowire and the Au particle are single crystalline and there is an overlap of both structures in the interface area as shown in Fig. 1c. The insert is the selected area diffraction (SAD) pattern of the Au particle along [112] orientation. Among these nanowires, some saw-shaped nanostructures were also observed. A typical nanosaw is exhibited in Fig. 2a. Fig. 2b reveals a SAD of the nanosaw along [126] orientation. Its corresponding high resolution TEM image is shown in Fig. 2c. Besides nanowires and nanosaws, $\mathrm{CdS}$ nanobelts were found on the $\mathrm{W}$ substrates placed in the temperature region of $600{ }^{\circ} \mathrm{C} \sim 750$ ${ }^{\circ} \mathrm{C}$. Some nanobelts have symmetric tips and some do not, as shown in Fig. 3a. In general, the body of a nanobelt is uniform. However, towards the end, the width of the belt gradually decreases along the growth direction and finally terminates in a thin tubular shape with a Au particle at the tip (Fig. 3b). An EDX line scan spectrum (Fig. 3c) was recorded from the tip area of a CdS nanobelt. It shows clearly that the Au particle contains CdS. This suggests that during the initial growth, evaporated $\mathrm{CdS}$ is carried by argon gas and dissolved into liquid $\mathrm{Au}$ particles. When the $\mathrm{Au}$ particle is supersaturated with $\mathrm{CdS}$, they starts to precipitate out of the Au particle at the liquid (Au)/solid (W) interface. After this initial stage, the direct diffusion of the $\mathrm{CdS}$ from the gas phase to the already formed nanobelt segment may also contribute to the further growth of the belt. A further study of this subject is in progress.

[1] X. F. Duan, Y. Huang, R. Agarwal, and C. M. Lieber, Nature 421 (2003) 241.

[2] L. F. Dong, J. Jiao, M. Coulter, and L. Love, submitted to Adv. Mater (January, 2003).

[3] R. S. Wagner and W. C. Ellis, Appl. Phys. Lett. 4 (1964) 89.

[4] L. F. Dong, J. Jiao, D. W. Tuggle, J. Petty, S. A. Elliff, and M. Coulter, Appl. Phys. Lett. 82 (2003) 1096.

[5] The authors would like to thank M. Coulter and T. Gushtyuk for helping with preparation of the samples. This research was supported by NSF under grant DMR-0097575, ECS-0217061, and an award from American Chemical Society Petroleum Research Fund (PRF-38108-G5). 

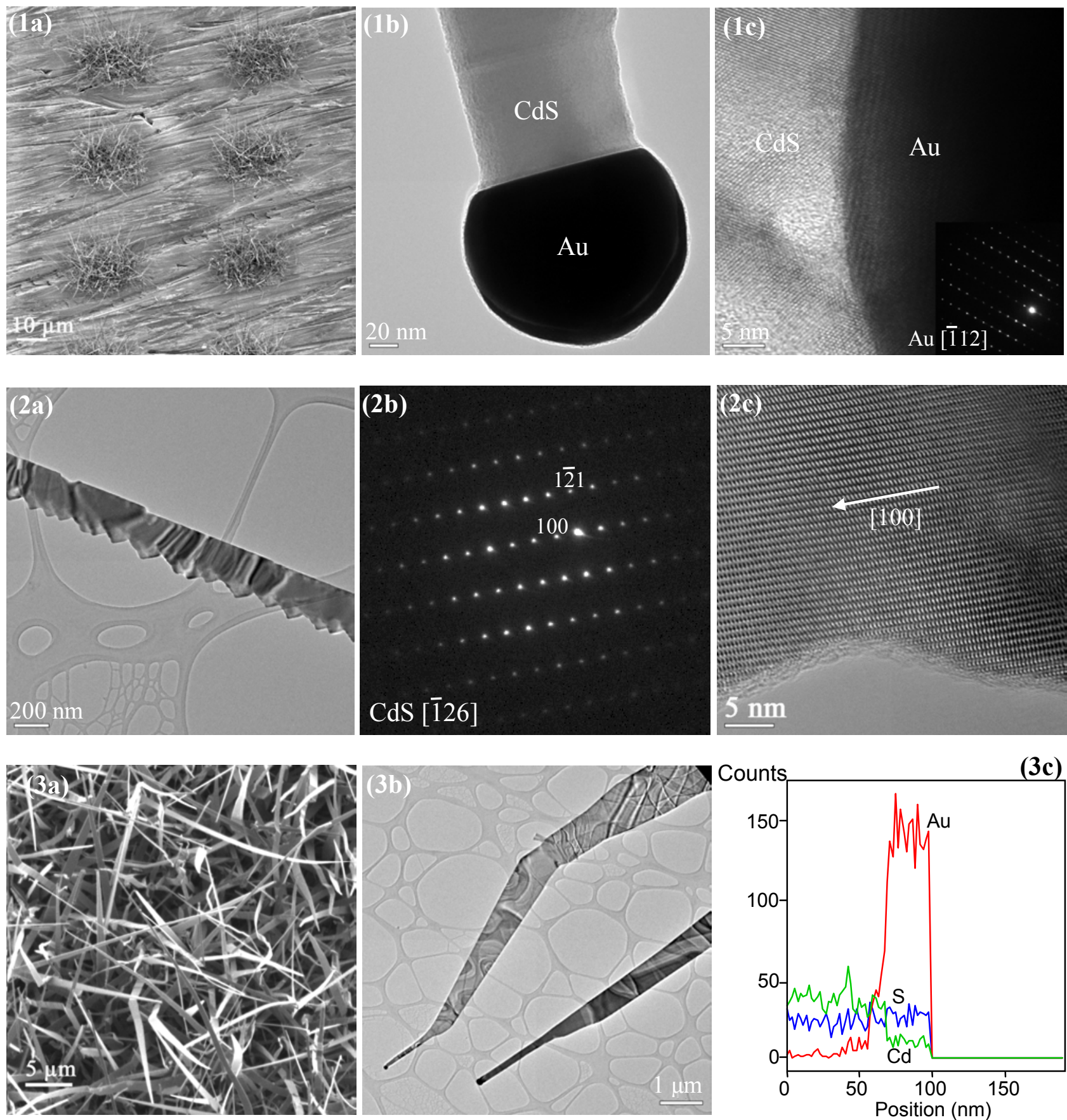

Counts

(3c)

Fig. 1 (a) SEM image of patterned growth of CdS nanowires. (b) TEM image of a Au particle located at the tip of a CdS nanowire; (c) HRTEM image of the interface between Au and CdS (Insert: SAD pattern of the Au particle).

Fig. 2 (a) TEM image of a saw-shaped CdS nanostructure. (b) SAD pattern and (c) HRTEM image of the nanosaw.

Fig. 3 (a) SEM image of CdS nanobelts, (b) TEM image of CdS nanobelts, (c) EDX line scan spectrum obtained from the tip area of a nanobelt. It shows that the line profiles of $\mathrm{Au}, \mathrm{Cd}$, and $\mathrm{S}$ overlap at the Au particle area. 